\title{
Severe infection may raise odds of autism in some children
}

\author{
BY GIORGIA GUGLIELMI
}

17 SEPTEMBER 2021

Severe infections during early childhood are linked to autism — at least in some boys, a new study in mice and people suggests. The findings were published today in Science Advances.

Researchers analyzed the health records of millions of children in the United States and found that boys diagnosed with autism are more likely than non-autistic boys to have had an infection requiring medical attention between age 1 and a half and 4 .

The study also showed that provoking a strong immune response in newborn mice with only one copy of TSC2, a gene tied to autism, leads to social memory problems in adult male rodents. In people, mutations in TSC2 cause tuberous sclerosis, a condition characterized by non-cancerous tumors and skin growths. About half of all people with tuberous sclerosis also have autism.

"If the TSC2 mutation was sufficient to cause autism, then everyone with that mutation would have autism - but they don't," says senior investigator Alcino Silva, director of the Integrative Center for Learning and Memory at the University of California, Los Angeles.

A child's chances of having autism rise with severe infections in the child or his mother, previous studies show, but not all children who contract serious infections go on to be diagnosed with autism.

The new study is the first to examine the relationship between immune activation and a specific genetic variant tied to autism, Silva says. The findings suggest that genetics and severe infection represent a 'two-hit' scenario for autism.

The work also highlights the importance of vaccinating children, says Manuel López Aranda, a postdoctoral fellow in Silva's lab. 


\section{Spectrum | Autism Research News}

https://www.spectrumnews.org

"If it's true that serious infections predispose kids to neuropsychiatric conditions, the best way to avoid serious infections in kids is to vaccinate them," he says.

\section{Immune activation:}

Silva, López Aranda and their colleagues injected newborn mice missing a copy of TSC2 with a compound that mimics a viral infection. When isolated from their mothers, the pups squeaked with shorter and simpler call sequences than did control pups, the researchers found. This simplified call repertoire may parallel early social communication difficulties in people with autism.

As adults, the male TSC2 mice seemed to have trouble recognizing and remembering other mice: They did not show the typical preference for spending time with a mouse they had never met before, but they did prefer a novel object over a familiar one.

An analysis of gene expression in three brain areas involved in social behavior suggested that, compared with controls, the male TSC2 mice injected with the viral mimic had increased levels of an immune molecule called interferon, which immune cells release to fight off viruses. The injection also activated microglia, immune cells in the brain that produce interferon and help to shape neuronal connections.

Sex differences in microglial number and function may explain why the immune activation led to social memory problems only in the male TSC2 mice, López Aranda says. The finding is in line with the observation that autism is about four times more prevalent in boys than it is in girls.

Depleting microglia or deleting the receptors for interferon rescued the animals' social memory problems. Giving the mice a drug called rapamycin, which is in trials for treating tuberous sclerosis, counteracted their strong immune activation and also relieved their social memory problems. Rapamycin acts by suppressing mTOR, a signaling pathway that is overactive in tuberous sclerosis and in response to viral infections.

To investigate whether immune activation in early childhood could be linked to social and other behavioral difficulties in people, the researchers looked at the insurance claims of more than 3.5 million children across the U.S. Of these, 4,417 girls and 18,232 boys had been diagnosed with autism. Autistic boys had a 40 percent increased likelihood of having contracted an infection requiring medical attention between the ages of 1 and half and 4 years, the researchers found.

\section{Two-hit model:}

Many researchers have long focused on either genetic or environmental factors that could contribute to autism. By looking at the interplay of both, the new study is "a breath of fresh air," says Mauro Costa-Mattioli, professor of neuroscience at Baylor College of Medicine in Houston, Texas, who was not involved in the research. 


\section{Spectrum | Autism Research News}

https://www.spectrumnews.org

The results of the animal experiments provide a mechanism by which immune response to viral infection could lead to some autism-like traits, Costa-Mattioli says. However, he cautions that the analysis of medical records reveals only an association between severe infection and autism diagnosis. This doesn't mean that the viral infection cause the behavioral difficulties associated with autism, he says.

It's also possible that the boys who contracted a serious infection and went on to be diagnosed with autism have genetic mutations linked to autism, says Eric Klann, director of the Center for Neural Science at New York University, who was not involved in the study. If that was true, Klann says, it would support the 'two-hit' hypothesis that the interaction between genes and the environment can increase autism risk.

The work may also have clinical implications in that it indicates that rapamycin eases the detrimental effects a strong immune activation may have on social skills, Silva says.

"Of course, this needs to be tested and demonstrated in humans," he says, "but our work suggests that it may just be possible."

Cite this article: https://doi.org/10.53053/MQUR6777 